\title{
Publicações científicas em ortopedia e traumatologia no Brasil: onde são concentradas e quais possíveis razões dessa concentração?
}

\section{Research in orthopedics and traumatology in Brazil: where are concentrated and what possible reasons for it?}

Sidney Wendell Goiana da Silva ${ }^{1}$. Giovanni de Andrade Lima ${ }^{2}$. Adriano de Almada Ferreira². Júlio César Chagas e Cavalcante ${ }^{3}$.

1 Discente do curso de Medicina, Universidade Federal do Ceará, Sobral, Ceará, Brasil. 2 Residente em Ortopedia de Traumatologia, Santa Casa de Misericórdia de Sobral, Sobral, Ceará, Brasil. 3 Mestre em Cirurgia, chefe do serviço de Ortopedia e Traumatologia da Santa Casa de Misericórdia de Sobral, professor do curso de Medicina, Universidade Federal do Ceará, Sobral, Ceará, Brasil.

\section{RESUMO}

Objetivo: mapear a realização de pesquisa em ortopedia no Brasil associando aos programas de pós-graduação credenciados, bem como o número de ortopedistas atuantes nas regiões verificadas. Metodologia: foi realizada uma análise descritiva das publicações nos dois principais periódicos na área, a Revista Brasileira de Ortopedia (RBO) e a Acta Ortopédica Brasileira (AOB), no período de 2011 a 2015, avaliando-se 836 publicações. Resultados: considerando-se os artigos nacionais, a região Sudeste predominou como principal sítio de publicações com 71,43\% dos artigos da RBO e 77,99\% das publicações da AOB. De outra forma, as regiões Nordeste e Norte demonstraram uma baixa participação em publicações da RBO, com 4,25\% e 1,16\% respectivamente. Já na AOB apenas a região Nordeste participou de publicações com 4,48\% dos artigos. A região Sudeste possui cerca de $70 \%$ dos programas de pós-graduação na área clínico-cirúrgica e/ou Ortopedia e Traumatologia. Conclusão: demonstra-se uma polarização das publicações no país, com maior número de pesquisas realizadas na região Sudeste, possivelmente relacionado ao maior número de programas de pós-graduação na área, associado ao grande número de profissionais na região.

Palavras-chave: Ortopedia. Publicações. Periódicos.

\section{ABSTRACT}

Aim: to map orthopedic research in Brazil associating to graduate and residency programs and number of orthopedic surgeons in the geographic regions as well. Method: a review of publications was performed in the both main journals, Revista Brasileira de Ortopedia (RBO) and Acta Ortopedica Brasileira (AOB) between 2011-2015, with a 836 publications analysis. Results: publications from national authors, southeast region was predominant in RBO $(71,43 \%)$ and AOB $(77,99 \%)$ papers. Otherwise, Northeast and North regions of Brazil demonstrated a low rate of publications in RBO, with 4,25\% and 1,16\% respectively. AOB showed publications from Northeast region only, with $4,48 \%$ of papers from this journal. Southeast region has $70 \%$ of graduate and residency programs in the country when Orthopedic surgery specialty is considered. Conclusion: orthopedical research is polarized in Brazil, where southeast region of the country is responsible for the most of publications in this field, possibly related to graduate residency programs existing in the region associated with larger number of practioners in orthopedics.

Keywords: Orthopedics. Publications. Periodicals.

Autor correspondente: Sidney Wendell Goiana da Silva, Av Comandante Maurocelio Rocha Pontes, 100, Derby, Sobral, Ceará. CEP: $62042-280$. Telefone: +55 88 3611-8000. E-mail: wendellgoianamed@gmail.com

Conflito de interesses: Não há qualquer conflito de interesses por parte de qualquer um dos autores.

Recebido em: 14 Ago 2016; Revisado em: 31 Jan 2017; Aceito em: 31 Jan 2017. 


\section{INTRODUÇÃO}

A pesquisa científica gera uma base de evidências seguras que auxilia o ortopedista na tomada de decisões esclarecidas, com o reconhecimento comprovado pelos mesmos. ${ }^{1}$ Entende-se que, resultados relevantes gerados a partir da pesquisa clínica tem importantes efeitos na melhoria da função e qualidade de vida dos pacientes, ${ }^{2,3}$ através de uma boa interpretação desses resultados gerados pelo ortopedista. ${ }^{4}$

No entanto, existe uma falta de envolvimento com a pesquisa que pode ser atribuída a vários fatores, tais como: falta de treinamento formal em metodologia de pesquisa, ${ }^{5}$ falta de conhecimento ${ }^{6}$ e restrição de tempo destinado à realização de trabalhos científicos. ${ }^{7}$

Para resolução do problema, enfatiza-se a necessidade da introdução de um programa de treinamento em pesquisa estruturado e funcional que possa auxiliar os cirurgiões ortopedistas a gerar hipóteses que levem efetivamente a publicações de alta qualidade. $^{8}$

A formação do ortopedista no Brasil compreende a atividade prática e teórica durante o período de 3 anos em um serviço reconhecido pela Sociedade Brasileira de Ortopedia e Traumatologia (SBOT) e pelo Ministério da Educação. ${ }^{9}$ Tradicionalmente, os métodos de pesquisa têm sido transmitidos através de cursos didáticos e seminários ou ainda são difundidos por meio da interação de mentores e aprendizes. Embora os pesquisadores ortopedistas iniciantes possam obter conhecimento teórico, as habilidades práticas são raramente transmitidas por esse método de ensino. ${ }^{8}$ No entanto, existem referencias que residentes envolvidos em pesquisas nos seus respectivos programas de treinamento possuem interesse futuro de se tornar pesquisadores. ${ }^{10}$

No último censo realizado, o Brasil possui cerca de 10.494 ortopedistas atuantes, sendo que a grande maioria está concentrada em grandes centros, e a maior parte dos estados da região Norte e Nordeste possuem menos que 100 especialistas. Em contraste, o Sudeste se apresenta como principal centro formador, possuindo mais de $60 \%$ dos programas de Residência Médica em Ortopedia e Traumatologia. ${ }^{11}$

Assim, o objetivo do estudo foi mapear a realização de pesquisa em ortopedia no Brasil, associando aos programas de pós-graduação médica devidamente credenciados e autorizados, bem como o número de ortopedistas atuantes nas regiões verificadas.

\section{METODOLOGIA}

Foi realizada uma análise das publicações nos dois principais periódicos da área de ortopedia e traumatologia, a Revista Brasileira de Ortopedia e a Acta Ortopédica Brasileira, ambas publicadas pela Sociedade Brasileira de Ortopedia e Traumatologia (SBOT), indexadas e classificadas na plataforma Qualis da Coordenação de Aperfeiçoamento de Pessoal de Nível Superior (CAPES) através da Plataforma Sucupira.
A análise consistiu da avaliação dos artigos, publicados no período de 2011 a 2015, através da base de dados Scielo. Os dados coletados foram: ano de publicação (volume e número), tipo de publicação (artigo de atualização, artigo de revisão, artigo original, relato de caso e nota técnica), região de origem do primeiro autor descrito no artigo (estado e cidade), bem como a unidade de realização da pesquisa descrita no artigo. Considerou-se para todos os parâmetros apenas os dados do primeiro autor.

Em associação, foi utilizado o relatório de Programas de Residência em Ortopedia e Traumatologia credenciadas à SBOT, para a relação entre unidades formadoras e volume de publicações. Adicionalmente, foi realizado uma associação entre o volume de publicações com o número de profissionais ortopedistas credenciados pelo Conselho Federal de Medicina. ${ }^{11}$

\section{RESULTADOS}

Os periódicos analisados consistem de periódicos classificados com nível B3 para o Acta Ortopédica Brasileira (AOB), e com nível B4 para a Revista Brasileira de Ortopedia (RBO), ambas na área de Medicina III (Plataforma Sucupira/CAPES, 2014).

Foram avaliados 545 artigos da RBO, sendo que 36 foram artigos de atualização (6,6\%), 17 artigos de revisão (3,11\%), 98 artigos de relato de caso $(17,98 \%)$, nove artigos de nota técnica $(1,65 \%)$ e 385 artigos originais $(70,64 \%)$. Do total de artigos, $519(95,22 \%)$ foram publicados por grupos nacionais enquanto que $26(4,78 \%)$ foram de contribuições de pesquisadores internacionais de países como Portugal, Estados Unidos, Colômbia e França.

Considerando-se os artigos nacionais, a região Sudeste, com $370(71,43 \%)$ artigos, predominou como o principal sítio de publicações nesse periódico, seguido pela região Sul com 90 (17,37\%), região Centro-Oeste com 30 (5,79\%), região Nordeste com $22(4,25 \%)$ e a região Norte com $6(1,16 \%)$ artigos (Gráfico 1).

Gráfico 1. Gráfico demonstrativo da porcentagem de publicações por região editados pela Revista Brasileira de Ortopedia - 2011 a 2015.

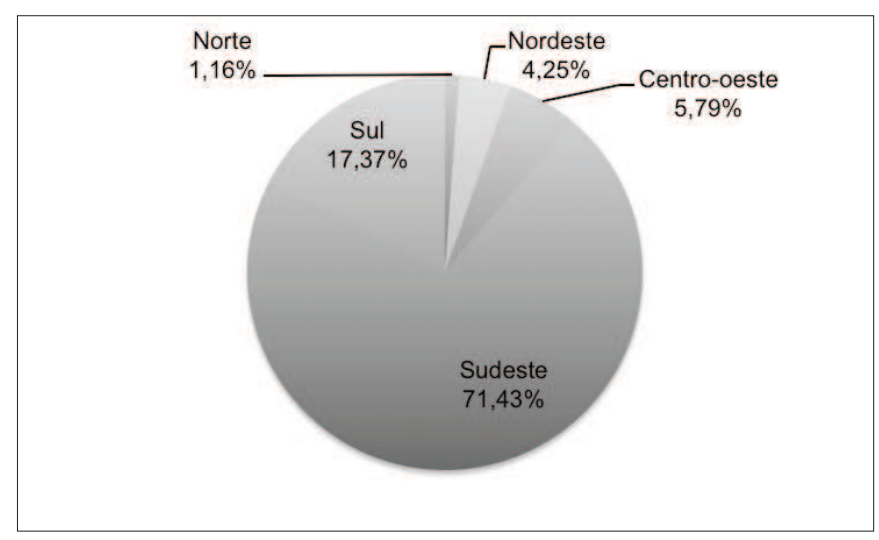


Das 27 unidades da federação, 17 estados foram representados em publicações da RBO (Gráfico 2), sendo que em 6 destes foram verificadas apenas uma única publicação no período analisado. Os resultados por estado estão demonstrados na Tabela 1.

Gráfico 2. Gráfico demonstrativo do volume de publicações em porcentagem por unidades federativas editados pela Revista Brasileira de Ortopedia - 2011 a 2015.

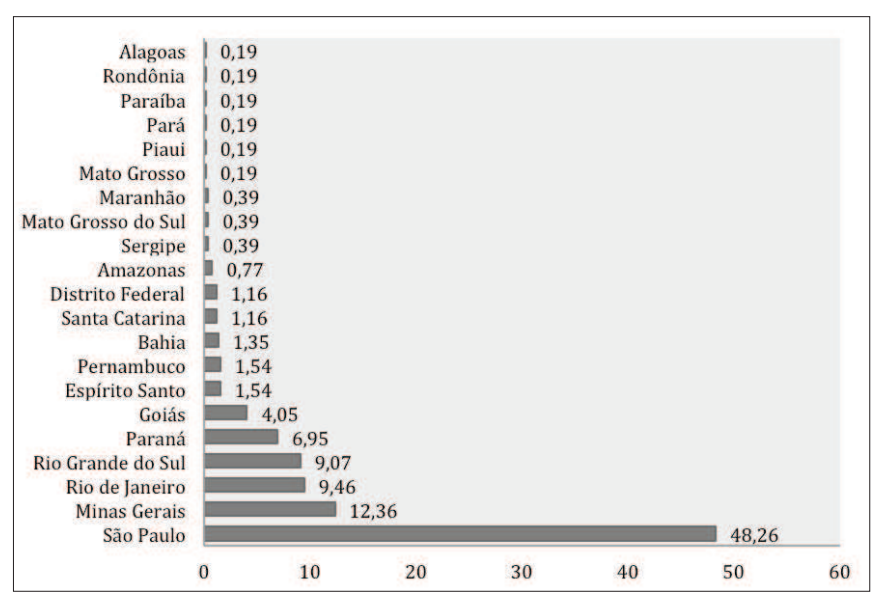

Tabela 1. Demonstrativo de publicações por estado nos periódicos Revista Brasileira de Ortopedia e Acta Ortopédica Brasileira - 2011 a 2015.

\begin{tabular}{|c|c|c|c|}
\hline \multirow[t]{2}{*}{ Estado } & \multicolumn{2}{|c|}{ Periódico } & \multirow[t]{2}{*}{ Total (\%) } \\
\hline & $R B O$ & $A O B$ & \\
\hline São Paulo & 250 & 189 & $439(55,92)$ \\
\hline Minas Gerais & 64 & 14 & $78(9,94)$ \\
\hline Rio de Janeiro & 49 & 10 & $59(7,52)$ \\
\hline Rio Grande do Sul & 47 & 6 & $53(6,75)$ \\
\hline Paraná & 36 & 15 & $51(6,50)$ \\
\hline Goiás & 21 & 4 & $25(3,18)$ \\
\hline Distrito Federal & 6 & 10 & $16(2,04)$ \\
\hline Bahia & 7 & 4 & $11(1,40)$ \\
\hline Espírito Santo & 8 & 3 & $11(1,40)$ \\
\hline Santa Catarina & 6 & 5 & $11(1,40)$ \\
\hline Pernambuco & 8 & 2 & $10(1,27)$ \\
\hline Amazonas & 4 & 0 & $4(0,51)$ \\
\hline Maranhão & 2 & 1 & $3(0,38)$ \\
\hline Ceará & 0 & 3 & $3(0,38)$ \\
\hline Mato Grosso do Sul & 2 & 0 & $2(0,25)$ \\
\hline Sergipe & 2 & 0 & $2(0,25)$ \\
\hline Alagoas & 1 & 0 & $1(0,13)$ \\
\hline Mato Grosso & 1 & 0 & $1(0,13)$ \\
\hline Pará & 1 & 0 & $1(0,13)$ \\
\hline Paraiba & 1 & 0 & $1(0,13)$ \\
\hline Piauí & 1 & 0 & $1(0,13)$ \\
\hline Rondônia & 1 & 0 & $1(0,13)$ \\
\hline Sergipe & 1 & 0 & $1(0,13)$ \\
\hline
\end{tabular}

Já da Acta Ortopédica Brasileira, foram avaliados 291 artigos, dos quais cinco foram artigos de atualização (1,7\%), 17 artigos de revisão (5,6\%), e 269 artigos originais (92,7\%). Não foram encontradas publicações referentes a relatos de caso ou notas técnicas. Da totalidade dos artigos, $25(8,6 \%)$ foram publicados por pesquisadores internacionais, de países como Cingapura, Turquia, Itália, Polônia, China, Egito, Nigéria, Portugal e Inglaterra, ao passo que $266(91,4 \%)$ artigos foram publicados por pesquisadores e instituições brasileiras.

A região Sudeste contribuiu com a publicação de 208 (77,99\%) artigos desse periódico, sendo seguida pela região Sul com 33 (12,31\%), região Centro-Oeste com $14(5,22 \%)$ e a região Nordeste com $12(4,48 \%)$ artigos. Não foram verificados artigos publicados com origem em estados da região Norte do Brasil no período estudado (Gráfico 3).

Dentre as unidades da federação, 14 estados foram responsáveis pelas publicações na $\mathrm{AOB}$, sendo que 6 destes foram representados com menos de cinco publicações durante todo o período (Gráfico 4).

Gráfico 3. Gráfico demonstrativo do volume de publicações em porcentagem por unidades federativas editados pela Revista Brasileira de Ortopedia - 2011 a 2015.

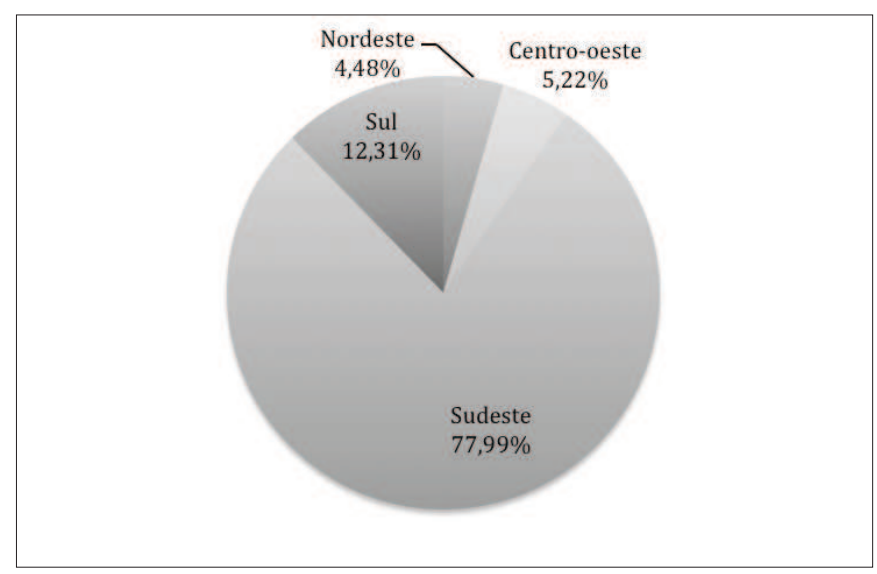

Gráfico 4. Gráfico demonstrativo do volume de publicações em porcentagem por unidades federativas editados pela Acta Ortopédica Brasileira no período de 2011 a 2015.

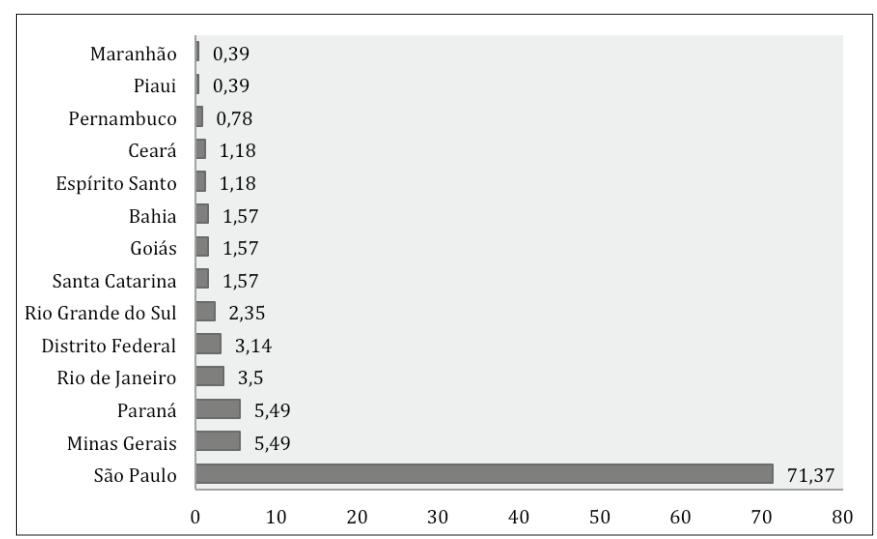




\section{DISCUSSÃO}

As tomadas de decisões baseadas em evidências são produzidas através das pesquisas clínicas, gerando resultados que influenciam na melhoria da função e qualidade de vida dos pacientes. ${ }^{2,3}$ A interpretação dos resultados, depende diretamente da publicação de pesquisas em periódicos. A Revista Brasileira de Ortopedia e a Acta Ortopédica Brasileira são os dois principais periódicos de pesquisa clínica e experimental na área de ortopedia e traumatologia no Brasil, sendo classificadas como Qualis B-4 e B-3, respectivamente. Essa classificação é realizada pelas áreas de avaliação e passa por um processo anual de atualização. Todos os veículos são enquadrados em estratos indicativos da qualidade em A1, o mais elevado, A2, B1, B2, B3, B4, B5 e C, sendo o último com peso zero. Assim, temos que os dois periódicos (RBO/AOB) avaliados encontram-se em uma faixa mediana de avaliação, pois este método afere a qualidade dos artigos e de outros tipos de produção, a partir da análise da indexação em bases de dados científicos e métrica do quantitativo de citações. ${ }^{12}$

Observou-se um bom envolvimento dos ortopedistas quanto à produção de pesquisa científica, com a publicação de um número expressivo de trabalhos agrupados nos periódicos avaliados, apesar de relatos na literatura demonstrarem que fatores como a falta de treinamento formal, ${ }^{5}$ falta de conhecimento em pesquisa ${ }^{6}$ ou restrição de tempo ${ }^{7}$ estarem relacionados com a falta de envolvimento dos mesmos na produção de conhecimento.

Observou-se uma polarização das publicações quando analisada a região do país de origem do artigo científico. A grande maioria das publicações, 587 artigos, teve origem na região Sudeste, o que provavelmente está relacionado ao grande número de ortopedistas atuantes nos estados que compõem o grupo. Além disso, a região Sudeste concentra a maior parte dos programas de residência médica em Ortopedia e Traumatologia, ${ }^{11}$ bem como os programas de pós-graduação stricto sensu (mestrado e doutorado) que contemplam pesquisas clínicas e experimentais.

De acordo com o portal Capes, ${ }^{13}$ existem 20 programas de pós-graduação stricto sensu diretamente relacionadas com áreas clínico-cirúrgicas e/ou ortopedia e traumatologia (área Medicina III), dos quais $70 \%$ estão localizados na região Sudeste, o que pode justificar o grande volume advindo desses estados. Isso está refletido no volume de publicações originadas dessa região tanto na RBO, com $71,43 \%$ dos artigos, quanto na AOB com 77,99\%.

Os outros programas estão localizados nas regiões Sul com três unidades, na região Nordeste com dois programas e a região Norte com um programa. A região Centro-Oeste não possui programa na área estudada cadastrado no portal Capes. ${ }^{13}$

\section{REFERÊNCIAS}

1. Bhandari M, Tornetta P 3rd, Guyatt GH. Glossary of evidence-based orthopaedic terminology. Clin Orthop Relat Res. 2003;(413):158-63.
A relação de publicação com números de profissionais associados a programas de pós-graduação stricto sensu é verdadeira nessa ordem, onde a região Sul foi a que mais contribuiu em publicação de artigos após a região Sudeste. Do total de publicações, 115 artigos tiveram origem de estados da região Sul.

No entanto, a região Centro-Oeste apesar de não possuir programas stricto sensu, contribuiu com a publicação de 44 artigos, sendo a terceira região que mais publicou nos periódicos analisados, o que pode estar relacionado com o número de profissionais atuantes na região, ${ }^{11}$ bem como a quantidade de programas de residência médica em ortopedia e traumatologia. ${ }^{13}$ A região Nordeste veio em seguida com 33 artigos, o que tem relação direta com um número reduzido de profissionais e programas de residência quando comparada às regiões anteriores. $\mathrm{O}$ mesmo conceito pode ser aplicado à região Norte, com um número total de publicações de seis artigos nos periódicos analisados. ${ }^{11}$

Ao analisar por estados, observou-se que 23 unidades federativas demonstraram publicações nos periódicos analisados. Apesar disso, foi demonstrado uma grande polarização das publicações, sendo concentradas no estado de São Paulo com uma participação de 55,92\% das publicações. Esse fato está diretamente relacionado ao conjunto que agrega um grande número de profissionais atuantes nesse estado, aliado ao grande número de programas de residência e programas de pós-graduação stricto sensu na área.

O estado de São Paulo abriga 40\% dos programas de mestrado e doutorado em áreas clínico-cirúrgicas e/ou ortopedia (Área Medicina III - Capes), sendo alguns específicos em pesquisa clínica e experimental na área de ortopedia e traumatologia, tais como os programas da Faculdade de Medicina de Ribeirão Preto (FMRP) e do Instituto de Ortopedia e Traumatologia do Hospital das Clínicas, ambos pertencentes à Universidade de São Paulo. ${ }^{13}$ Adicionalmente, o estado contempla um grande número de programas de residência, o que estimula a publicação em pesquisa clínica e observacional. ${ }^{11}$

\section{CONCLUSÕES}

Há envolvimento dos ortopedistas na publicação de conhecimento clínico e experimental, demonstrado pelo número de artigos nos periódicos analisados. No entanto, ainda se observa uma polarização das publicações, sendo concentradas na região Sudeste do país, mais especificamente no estado de São Paulo, provavelmente pelo número de profissionais atuantes, associado ao maior número de programas de residência e programas de pós-graduação stricto sensu na área.

\section{CONFLITOS DE INTERESSE}

Os autores declaram não haver conflitos de interesse.
2. Szabo RM. Outcomes assessment in hand surgery: when are they meaningful? J Hand Surg Am. 2001;26(6):993-1002. 
3. Higgins JP, Green S, editors. Cochrane handbook for systematic reviews of interventions version 5.1.0 [Internet]. [S. 1.]: The Cochrane Collaboration; 2011 [acesso em 2016 jul 15]. Disponível em: www. handbook.cochrane.org

4. Moraes VY, Ferrari PM, Gracitelli GC, Faloppa F, Belloti JC. Outcomes in orthopedics and traumatology: translating research into practice. Acta Ortop Bras. 2014;22(6):330-3.

5.Seida JC, LeBlanc C, Schouten JR, Mousavi SS, Hartling L, Vandermeer B, et al. Systematic review: nonoperative and operative treatments for rotator cuff tears. Ann Intern Med. 2010;153(4):24655 .

6. Moraes VY, Belloti JC, Faloppa F, Bhandari M. Collaborative multicenter trials in Latin America: challenges and opportunities in orthopedic and trauma surgery. Sao Paulo Med J. 2013;131(3):187-92.

7. Williamson PR, Gamble C, Altman DG, Hutton JL. Outcome selection bias in meta-analysis. Stat Methods Med Res. 2005; 14(5):515-24.

8. Ferreira AP, Rajgor D, Shah J, Shah A, Pietrobon R. Treinamento em pesquisa global em cirurgia ortopédica: semente para uma rede internacional. Acta Ortop Bras. 2012;20(2):110-7.
9. Karam FC, Lopes MH. Ortopedia: origem histórica, o ensino no Brasil e estudos metodológicos pelo mundo. Sci Med. 2005;15(3):172-8.

10. Ahn J, Watt CD, Greeley SA, Bernsteins J. MD-PhD students in a major training program show strong interest in becoming surgeonscientists. Clin Orthop Relat Res. 2004;(425):258-63.

11. Scheffer M, coordenador. Demografia Médica no Brasil, v. 2 [Internet]. São Paulo: Conselho Regional de Medicina do Estado de São Paulo, Conselho Federal de Medicina; 2013 [acesso em 2016 jul 15]. 258 p. Disponível em: http://www.cremesp.org.br/pdfs/ DemografiaMedicaBrasilVol2.pdf

12. Capes. Classificação da Produção Intelectual [Internet]. [atualizado em 01 ago. 2016; acesso em 2016 jul 15]. Disponível em: http://www.capes.gov.br/avaliacao/instrumentos-de-apoio/ classificacao-da-producao-intelectual

13. Capes. Tabela de Áreas de Conhecimento/Avaliação [Internet]. Brasília, Capes; 2014 [atualizado em 2017 jan 31; acesso em 2016 jul 15]. Disponível em: http://www.capes.gov.br/avaliacao/ instrumentos-de-apoio/tabela-de-areas-do-conhecimento-avaliacao

\section{Como citar:}

Silva SW, Lima GA, Ferreira AA, Cavalcante JC. Publicações científicas em ortopedia e traumatologia no Brasil: onde são concentradas e quais possíveis razões dessa concentração? Rev Med UFC. 2018 jan-mar;58(1):8-12. 